\title{
Sequencing down the line
}

A popular sequencing platform can add long-range information to short sequence reads.

For curious minds, a sealed gadget is never safe. So it was natural for Jerrod Schwartz and Jay Shendure to pop open the hood of their Illumina GAIIx sequencer. With their colleagues at the University of Washington, Seattle, they wanted to overcome the limitations of short read lengths generated by nextgeneration sequencers. Short reads can be difficult to overlap during genome assembly and often fail to reveal structural genomic variation. By manipulating DNA after loading it on the machine, the group found a way to associate different short reads with much longer single molecules.

The Illumina platform works by anchoring the two ends of a DNA fragment to a surface, forming a bridge that can be amplified to create a DNA cluster. Discrete clusters are then sequenced using a fluorescence-based visual readout. The process offers efficiency and throughput but does not work on long molecules. "The cost per base is no longer the sole arbiter in terms of enabling technologies," says Shendure. He argues that contiguity information-knowing the order or spacing of short reads - can overcome many difficulties in genomic analysis.

In their proof-of-principle work, the researchers tethered both ends of large bacterial DNA fragments bearing a single primer sequence to a GAIIx flow cell. They then applied an electric field using homemade electrodes to stretch the DNA out. To generate fragments short enough for bridge amplification, they added an efficient transposase that inserted a second primer near the DNA ends. By visualizing the sequence from the ends of molecules prepared directly on the flow cell, the team could use the distance between clusters as an estimate of distance on a single molecule.
The method worked reasonably well for fragments up to 8 kilobases. By comparison, paired-end sequencing works well up to 3 kilobases; 40-kilobase fosmid approaches exist but require more involved preparation. The goal is to look at still longer molecules and to create several internal sequencing sites along the linear molecules. Anchoring and stretching long molecules is tricky, says Schwartz, and the group is also working out other technical issues.

In the future, single-molecule sequencing methods may make long-range information readily available, but regardless, enhancing a well-used instrument for the purpose certainly has appeal.

\section{Tal Nawy}

\section{RESEARCH PAPERS}

Schwartz, J.J. et al. Capturing native long-range contiguity by in situ library construction and optical sequencing. Proc. Natl. Acad. Sci. USA 109, 1874918754 (2012) 